\title{
Autopercepção do paciente com doença pulmonar obstrutiva crônica sobre seu desempenho ocupacional em tempo de pandemia de COVID-19 1
}

\section{Self-perception of the patient with chronic obstructive pulmonary disease on its occupational performance during COVID-19 pandemic}

Roberta Fontenele de Vasconcelos ${ }^{\mathrm{a}}$ (D), Marília Ximenes Freitas Frota ${ }^{\mathrm{b}}$ (D), Valéria Barroso de Albuquerque ${ }^{c}$ (D), Marilene Calderaro da Silva Munguba ${ }^{\mathrm{d}}$ (i)

${ }^{a}$ Universidade de Pernambuco - UPE, Recife, PE, Brasil.

${ }^{\text {b} U n i v e r s i d a d e ~ E s t a d u a l ~ d o ~ C e a r a ́ ~-~ U E C E, ~ F o r t a l e z a, ~ C E, ~ B r a s i l . ~}$

'Universidade de Fortaleza - UNIFOR, Fortaleza, CE, Brasil.

${ }^{\mathrm{d} U n i v e r s i d a d e ~ F e d e r a l ~ d o ~ C e a r a ́ ~-~ U F C, ~ F o r t a l e z a, ~ C E, ~ B r a s i l . ~}$

Como citar: Vasconcelos, R. F., Frota' M. X. F., Albuquerque, V. B., \& Munguba, M. C. S. (2022). Autopercepção do paciente com doença pulmonar obstrutiva crônica sobre seu desempenho ocupacional em tempo de pandemia de COVID-19. Cadernos Brasileiros de Terapia Ocupacional, 30, e2983.

https://doi.org/10.1590/2526-8910.ctoAO22612983

\begin{abstract}
$\underline{\text { Resumo }}$
Introduçáo: A Doença Pulmonar Obstrutiva Crônica (DPOC) é caracterizada pela limitação progressiva do fluxo aéreo. Os pacientes também desenvolvem manifestaçóes sistêmicas que determinam o declínio progressivo da capacidade funcional. Objetivo: Conhecer a autopercepção do paciente com DPOC sobre seu desempenho ocupacional destacando a influência da pandemia de COVID-19 no seu cotidiano. Método: Pesquisa de natureza descritiva e abordagem qualitativa, tendo como técnica a análise de conteúdo na modalidade temática. A amostra foi constituída de nove pacientes com DPOC atendidos no programa de manutenção pós-reabilitaçáo pulmonar em hospital de referência de saúde em cardiopneumologia. A coleta dos dados ocorreu de junho a agosto de 2020, mediante roteiro de entrevista semiestruturada, por meio de chamadas telefônicas. As entrevistas gravadas foram transcritas e submetidas à análise do conteúdo, por meio da análise temática. Resultados: As ocupações cotidianas percebidas com dificuldade de serem realizadas em decorrência da DPOC foram sono, trabalho, participação social, lazer, além de Atividades de Vida Diária (AVD) - mobilidade
\end{abstract}

${ }^{1}$ Pesquisa integrante do trabalho de conclusão da Residência Integrada em Saúde da Escola de Saúde Pública do Ceará (ESP-CE) do componente hospitalar e ênfase em Cardiopneumologia. Este estudo foi aprovado pelo Comitê de Ética em Pesquisa do Hospital Dr. Carlos Alberto Studart Gomes sob parecer de n³.927.816 e CAAE nº 29585520.6.0000.5039. Todos os pacientes expressaram seu consentimento em participar da pesquisa.

Recebido em Mar. 8, 2021; 1ª Revisão em Jul. 8, 2021; Aceito em: Out. 4, 2021 
funcional, banho, higiene pessoal, vestir e atividade sexual - e Atividades Instrumentais da Vida Diária (AIVD) - gerenciamento do lar e fazer compras. As ocupaçóes limitadas pela DPOC foram ainda mais impactadas pelas medidas restritivas causadas pela COVID-19. Conclusão: Com base na compreensão das dificuldades do paciente com DPOC em realizar suas ocupaçôes, foi possível conhecer o quanto a DPOC repercute no desempenho ocupacional e interfere nas mais diversas ocupaçóes que trazem significado e propósito à vida. A pandemia de COVID-19 limitou ainda mais o envolvimento do paciente com DPOC nas suas ocupaçóes significativas.

Palavras-chave: Doença Pulmonar Obstrutiva Crônica, Atividades Cotidianas, COVID-19, Terapia Ocupacional.

\section{$\underline{\text { Abstract }}$}

Introduction: Chronic Obstructive Pulmonary Disease (COPD) is characterized by progressive airflow limitation. In addition patients with COPD develop systemic manifestations that determine the progressive decline in functional capacity. Objective: To know the self-perception of patients with COPD about their occupational performance, also highlighting the influence of the COVID-19 pandemic on their daily lives. Method: Research of a descriptive nature and qualitative approach, as a technique the analysis of content in the thematic modality. The sample consisted of 9 COPD patients seen in the post-pulmonary rehabilitation maintenance program at a reference hospital in the provision of health services in cardiopneumology. Data collection took place from June to August 2020, through semi-structured interview script, through telephone calls. The recorded interviews were transcribed and submitted to content analysis, through the thematic analyses. Results: Daily occupations perceived as difficult to perform as a result of COPD are part of the category sleep, work, social participation, leisure, Activities of Daily Living (ADL) functional mobility, bathing, personal hygiene, dressing and sexual activity - and Instrumental Activities of Daily Living (IADL) - home management and shopping. Occupations limited by COPD were further impacted by the restrictive measures caused by COVID-19. Conclusion: Based on the understanding of the difficulties of COPD patients in performing their occupations, it was possible to know how much COPD has an impact on occupational performance and interferes in the most diverse occupations that bring meaning and purpose to life. The COVID-19 pandemic further delimited the involvement of patients with COPD in their significant occupations.

Keywords: Pulmonary Disease, Chronic Obstructive, Activities of Daily Living, COVID-19, Occupational Therapy.

\section{Introduçáo}

A Doença Pulmonar Obstrutiva Crônica (DPOC) é uma enfermidade evitável e tratável, caracterizada pela limitação do fluxo aéreo que não é totalmente reversível. Essa limitação do fluxo aéreo geralmente é progressiva e associada a uma resposta inflamatória anormal do pulmão a partículas e/ou gases nocivos, sendo o 
tabagismo o principal fator de risco (Global Initiative for Chronic Obstructive Lung Disease, 2021).

Atualmente, a DPOC é uma das principais causas de morbidade crônica e a terceira principal causa de morte no mundo, responsável por 3,2 milhóes de mortes em 2017, com estimativa do aumento progressivo deste número nos próximos anos (Roth et al., 2018). No Brasil, ela ocupa a terceira causa de morte entre as doenças crônicas não transmissíveis (Rabahi, 2013). A DPOC continuará sendo um problema de saúde pública significativo no futuro devido à exposição contínua a fatores de risco e ao envelhecimento da população (Rabe \& Watz, 2017; Global Initiative for Chronic Obstructive Lung Disease, 2021).

A disfunção pulmonar, as manifestações sistêmicas e o aumento da dispneia podem conduzir à piora progressiva do condicionamento físico, intolerância ao exercício físico e à inatividade dos pacientes com DPOC, determinando o declínio progressivo da capacidade funcional impactando no desempenho ocupacional (Kim et al., 2008; Silva et al., 2017).

A dispneia, caracterizada pela dificuldade de respirar, é o sintoma que mais gera limitação. Os pacientes com maior queixa de cansaço apresentam pior condição de realizar as atividades do cotidiano. Quanto maior a sensação de falta de ar, mais preponderante será sua limitação e restrição em realizar as atividades cotidianas (Silva et al., 2015).

Conforme Machado (2018), a progressão das doenças pulmonares leva a uma piora da sintomatologia, ocasionando limitação progressiva das atividades do cotidiano. Essa limitação pode se tornar um ciclo vicioso e evoluir até a dependência funcional do indivíduo, alterando sua vida social, econômica e emocional, fatores que podem interferir na sua qualidade de vida.

Assim, é necessário que as condutas terapêuticas oportunizadas ao paciente com DPOC não só mensurem objetivamente o desempenho, as respostas fisiológicas e a dispneia no momento em que realizam as atividades do cotidiano, mas que também seja considerado o impacto que essas dificuldades e limitaçóes causam nas suas ocupaçóes (Gulart et al., 2015).

As ocupações se referem às atividades cotidianas que as pessoas realizam como indivíduos, nas famílias e nas comunidades, para ocupar o tempo e trazer significado e propósito à vida; elas incluem as atividades que as pessoas precisam, querem e devem fazer (World Federation of Occupational Therapy, 2020).

A terapia ocupacional tem como princípio os elementos chaves do desempenho ocupacional com propósitos que habilitam e permitem os pacientes a desempenharem suas tarefas significativas, possibilitando dessa forma satisfação pessoal e bem-estar, minimizando as dificuldades e limitaçóes para desempenhar suas ocupaçóes em decorrência de sua doença. Encoraja-se o paciente a se envolver ativamente durante todo o processo, com a finalidade de alcançar objetivos que sejam significativos e relevantes para a sua vida diária (Hagedorn, 2003).

O desempenho das suas ocupaçóes deve ser determinado pelo próprio paciente com base em suas experiências. Ninguém melhor do que ele para determinar como desempenha suas ocupações diárias (Caldas et al., 2011). Cabe ao terapeuta ocupacional conhecer a percepção do cliente sobre seu desempenho ocupacional e utilizar como guia baseado na prática centrada no cliente. Conforme a American 
Occupational Therapy Association (2020), ao utilizar uma abordagem centrada no cliente, o profissional de terapia ocupacional reúne informaçóes para entender o que, naquele momento, é importante e significativo ao paciente.

$\mathrm{O}$ interesse por essa pesquisa surgiu durante a vivência da pesquisadora como terapeuta ocupacional residente em um hospital de referência no atendimento aos pacientes com DPOC, ao perceber a necessidade de se refletir sobre o desempenho ocupacional que faz parte da rotina desses pacientes, assim como a influência da pandemia de COVID-19 nessas ocupaçóes.

Essa pesquisa se justifica pela necessidade percebida durante os atendimentos aos pacientes com DPOC de proporcionar espaço e foco aos seus aspectos relacionais e significativos, buscando compreender a autopercepção do paciente com DPOC em relação ao seu desempenho ocupacional. Por ter sido realizada no período da pandemia de COVID-19, foi possível destacar também a influência da interrupção e privação das ocupações nesse momento. A partir disto, o objetivo da presente pesquisa foi conhecer a autopercepção do paciente com DPOC sobre seu desempenho ocupacional, destacando também a influência da COVID-19 no seu cotidiano.

\section{Método}

\section{Tipo de pesquisa}

Trata-se de um estudo de natureza descritiva com abordagem qualitativa. De acordo com Minayo (2014), o estudo qualitativo lida com o universo de significados, crenças, valores e atitudes, o que se adequa a um espaço mais profundo das relaçóes, dos processos e dos fenômenos que não podem ser reduzidos a operacionalizaçóes de variáveis, favorecendo a interpretação de particularidades dos comportamentos e atitudes individuais, tendo como técnica a análise de conteúdo na modalidade temática (Bardin, 2009).

\section{Local da pesquisa}

O estudo foi realizado no Hospital Dr. Carlos Alberto Studart Gomes (Hospital de Messejana), especializado no diagnóstico e tratamento de doenças cardíacas e pulmonares, da cidade de Fortaleza-CE, centro de referência na prestação de serviços de saúde em cardiologia e pneumologia da Regiáo Norte e Nordeste.

\section{Participantes da pesquisa}

A população do estudo foi composta pelos pacientes com DPOC que participam do programa de manutenção pós-reabilitação pulmonar, o qual é denominado "Caminhada do Bosque" - programa idealizado com o objetivo de manutenção dos benefícios alcançados após alta da reabilitação pulmonar, evitando a redução da perda funcional causada pela doença pulmonar e o favorecimento da otimização das atividades físicas, emocionais e sociais, 
proporcionando ao paciente a maximização e manutenção da sua independência e autonomia.

A amostra foi constituída por 9 (nove) pessoas de ambos os sexos, com idade entre 57 e 78 anos. Os entrevistados foram identificados por meio de números de acordo com a ordem da entrevista, de modo que a real identidade de cada participante fosse preservada. Utilizou-se P1 para o paciente número 1, seguindo sucessivamente a numeração necessária até o P9, a quantidade de pacientes entrevistados. Eles foram selecionados de acordo com os seguintes critérios de inclusão: diagnóstico clinico de DPOC, independente do estadiamento da doença; alta recebida do programa de reabilitaçáo pulmonar; e participação frequente no programa de manutenção pós-reabilitação pulmonar, o "Caminhada do Bosque". Para os critérios de exclusão, foi considerado o comprometimento auditivo grave, o que certamente impediria a compreensão dos objetivos e participação no presente estudo, assim também como o telefone temporariamente desligado ou fora da área de cobertura durante as tentativas de contato.

\section{Coleta de dados}

A coleta de informaçóes ocorreu durante o período de junho a agosto de 2020. Os dados correspondentes às ocupaçóes foram obtidos de forma qualitativa mediante a utilização de roteiro de entrevista semiestruturada, por meio de chamadas telefônicas devido à suspensão dos atendimentos presenciais em decorrência da pandemia da COVID-19.

As entrevistas gravadas foram transcritas e submetidas à análise do conteúdo, por meio da modalidade temática de Bardin, que tem como objeto a superação da incerteza, confirmando ou não o que se julga ver na mensagem. A leitura do material leva à validação e à generalização dos dados, enriquecendo a leitura pela descoberta de conteúdos e estruturas que confirmam ou negam o que se procura demonstrar, a propósito das mensagens e elementos de significação (Bardin, 2009).

Foram realizadas as transcrições na íntegra com a preservação da máxima fidelidade entre o diálogo e o texto escrito, o que possibilitou à pesquisadora perceber a dimensão do impacto que a DPOC causa na vida dos sujeitos e como foi deixarem de realizar suas ocupaçóes significativas no período da pandemia, além dos sentimentos intrínsecos e outros significados que surgiram.

\section{Consideraçóes éticas}

Em se tratando de aspectos éticos que subsidiaram o estudo, foram respeitadas as exigências da resolução 466/12 do Conselho Nacional de Saúde, que dispóe sobre as normas e diretrizes regulamentadoras de pesquisas envolvendo os seres humanos. Este estudo foi aprovado pelo Comitê de Ética em Pesquisa do Hospital Dr. Carlos Alberto Studart Gomes, sob parecer de no 3.927.816 e CAAE no 29585520.6.0000.5039. Todos os pacientes presentes na amostra expressaram seu 
consentimento em participar da pesquisa por meio do consentimento livre e esclarecido informado por telefone no primeiro contato.

\section{Análise dos dados}

Para alcançar mais precisamente os significados manifestos e latentes trazidos pelos pacientes os dados, eles foram submetidos à análise de conteúdo de Bardin (2009), destacando as unidades de significados, as categorias temáticas e ideias essenciais.

A trajetória de análise dos discursos dos pacientes com DPOC sobre sua autopercepção do desempenho ocupacional aconteceu inicialmente com a transcrição das entrevistas gravadas. Após a leitura, foram realizadas as reduçóes independentes, alcançadas por zonas de convergência entre as falas dos pacientes, das quais foram retiradas as unidades de significados, iniciando a condução da codificação, que corresponde à transformação dos dados brutos em categorias temáticas, permitindo uma descrição das características relevantes do conteúdo.

As categorias temáticas encontradas foram: mobilidade funcional, banho, higiene pessoal, vestir-se, atividade sexual, estabelecimento e gerenciamento do lar, fazer compras, sono, participação social, trabalho, lazer e COVID-19.

Após o reconhecimento das dificuldades na realização das ocupaçóes cotidianas do paciente com DPOC e do rompimento das mesmas na pandemia, compreenderam-se as ideias essenciais: percepção do paciente com DPOC sobre suas atividades cotidianas e repercussóes da pandemia por COVID-19 no cotidiano do paciente com DPOC.

\section{Resultados e Discussão}

Os resultados foram apresentados juntamente com as discussôes advindas das entrevistas realizadas com os pacientes com DPOC. A partir da análise das entrevistas, surgiram os temas que mais se destacaram dentre os discursos e orientaram a construção das seguintes ideias essenciais: percepção do paciente com DPOC sobre suas atividades cotidianas e repercussóes da pandemia por COVID19 no cotidiano do paciente com DPOC.

\section{Percepçáo do paciente com DPOC sobre suas atividades cotidianas}

O percurso para compreender a autopercepção do paciente com DPOC sobre seu desempenho ocupacional, a partir do processo desenvolvido neste estudo, encontra-se diante de inúmeras possibilidades, incluindo a percepção relatada sobre as suas atividades cotidianas.

As Atividades de Vida Diária (AVD) realizadas com dificuldade em decorrência da DPOC mencionadas pelos pacientes fazem parte das seguintes categorias: mobilidade funcional, banho, higiene pessoal, vestir-se e atividade sexual, repercutindo no seu desempenho em realizar as tarefas da vida diária e limitando a qualidade da performance, assim como explicitam as falas a seguir (Tabela 1). 
Tabela 1. Unidades de significado mencionadas nas AVD.

\begin{tabular}{|c|c|}
\hline Atividades de Vida Diária (AVD) & Contexto das Unidades de Significado \\
\hline \multirow{5}{*}{ Mobilidade funcional } & "Ao andar, eu canso" (P1) \\
\hline & "Sinto dificuldade de subir escada" (P4) \\
\hline & "Subir e descer escada é um problema" (P6) \\
\hline & "Subir a escada é um sufoco" (P7) \\
\hline & "Não consigo caminhar carregando sacola" (P9) \\
\hline \multirow{7}{*}{ Banho } & $\begin{array}{l}\text { "Sinto dificuldade de levantar os braços para lavar os cabelos e baixar para passar } \\
\text { a escovinha nos pés" (P1) }\end{array}$ \\
\hline & "Vou tomar banho e canso" (P2) \\
\hline & $\begin{array}{l}\text { "A água do chuveiro cai na minha cabeça e fico com falta de ar; canso quando } \\
\text { faço um banho mais demorado" (P5) }\end{array}$ \\
\hline & "No banho, eu canso" (P6) \\
\hline & "Termino o banho já cansada" (P7) \\
\hline & "Banho só com o cateter nasal" (P8) \\
\hline & "Termino de tomar banho cansada" (P9) \\
\hline \multirow{3}{*}{ Higiene Pessoal } & $\begin{array}{l}\text { "Gostava muito de cuidar das minhas unhas, usar o alicate; não consigo abaixar } \\
\text { para poder fazer; quem fazia era eu" (P1) }\end{array}$ \\
\hline & "Náo consigo pentear o cabelo, mas, com o aparelho (cateter nasal), eu faço" (P8) \\
\hline & "Lavar o cabelo eu canso" (P10) \\
\hline \multirow{5}{*}{ Vestir-se } & "Se baixar para me calçar, canso" (P2) \\
\hline & "Sinto dificuldade de abotoar o sutiā." (P3) \\
\hline & "Me sinto cansada ao vestir a roupa, com falta de ar" (P5) \\
\hline & "Vestir roupa apertada eu fico cansada, abotoar um sutiã" (P9) \\
\hline & "Calçar o sapato cansa. (P10) \\
\hline \multirow{3}{*}{ Atividade sexual } & $\begin{array}{l}\text { "Mudou drasticamente a minha vida conjugal; a parte da relaçáo sexual é muito } \\
\text { difícil para mim. " (P3) }\end{array}$ \\
\hline & "Namorar é devagarinho; tem que ser com calma" (P7) \\
\hline & "O marido também tem as limitações dele; aí dá certo. " (P9) \\
\hline
\end{tabular}

Fonte: Dados da pesquisa, 2020.

O documento oficial, Occupational therapy practice framework: Domain and process, da Associação Americana de Terapia Ocupacional (American Occupational Therapy Association, 2020), o qual descreve a estrutura e o domínio da prática da terapia ocupacional, aponta que as atividades de vida diária (AVD) fazem parte do domínio ocupaçóes e as descreve como as atividades orientadas para o cuidado do paciente com o seu próprio corpo, sendo fundamentais para viver no mundo social, permitindo a sobrevivência básica e o bem-estar.

Pacientes com DPOC frequentemente experimentam problemas durante o desempenho das AVD. A dispneia é frequentemente relatada como fator limitante para o desempenho das ocupaçóes. Os pacientes com DPOC experimentam um maior grau de dispneia durante as AVD em comparação com pessoas saudáveis, mesmo nos estágios iniciais da doença. A realização dessas atividades aumenta as demandas ventilatórias e induzem a hiperinsuflação dinâmica em pacientes com DPOC (Vaes et al., 2011; Vaes et al., 2019).

Conforme a resolução do Conselho Federal de Fisioterapia e Terapia Ocupacional- Conselho Federal de Fisioterapia e Terapia Ocupacional (Brasil, 2006) no 316, de 19 de julho de 2006, é de exclusiva competência do terapeuta ocupacional, no âmbito de sua atuação, avaliar as habilidades funcionais do indivíduo, elaborar 
a programação terapêutico-ocupacional e executar o treinamento das funçóes para o desenvolvimento das capacidades de desempenho das Atividades de Vida Diária (AVD) e Atividades Instrumentais de Vida Diária (AIVD).

As atividades instrumentais de vida diária (AIVD) também fazem parte do domínio ocupação humana e são caracterizadas por serem as atividades de apoio à vida diária dentro de casa e na comunidade, as quais necessitam de interações mais complexas que as utilizadas nas AVD (American Occupational Therapy Association, 2020).

As AIVDs realizadas com dificuldade em decorrência da DPOC mencionadas pelos pacientes fazem parte das seguintes categorias: estabelecimento e gerenciamento do lar e o fazer compras (Tabela 2).

Tabela 2. Unidades de significado mencionadas nas AIVD.

\begin{tabular}{ll}
\hline Atividades Instrumentais de Vida Diária (AIVDs) & Contexto das Unidades de Significado \\
\hline & "Náo varro mais a casa" (P1) \\
\hline Estabelecimento e gerenciamento do lar & "Dificuldade em estender a roupa" (P3) \\
& "Dificuldade de varrer casa" (P6) \\
& "Lavo na mão uma peça pequena" (P7) \\
& "Não lavo roupa; varrer a casa, tem que tirar tudo do meio" (P8) \\
\hline "Tenho dificuldade de varrer, passar o pano" (P9) \\
\hline Fazer compras & "Não faço mais compra sozinha" (P5) \\
\hline & "Nunca vou sozinha fazer compra; não consigo empurrar o \\
& carrinho' (P7) \\
\hline
\end{tabular}

Fonte: Dados da pesquisa, 2020.

Além das AVD e AIVD, outras atividades cotidianas que fazem parte da rotina dos pacientes com DPOC foram relatadas, como o sono, participação social, trabalho e lazer (Tabela 3).

Tabela 3. Unidades de significado mencionadas em outras atividades cotidianas.

\begin{tabular}{|c|c|}
\hline Outras Ocupaçóes & Contexto das Unidades de Significado \\
\hline \multirow{2}{*}{ Sono } & "A tosse me acorda e demoro a dormir" (P5) \\
\hline & "Não durmo bem, me sinto sufocar" (P7) \\
\hline \multirow[b]{2}{*}{ Participação social } & "Não vou mais para meu grupo da igreja; não consigo ajudar nas funçôes" (P6) \\
\hline & $\begin{array}{l}\text { "Depois que comecei a usar o oxigênio, não saio mais de ônibus, quase não saio para casa dos } \\
\text { familiares" (P8) }\end{array}$ \\
\hline \multirow{3}{*}{ Trabalho } & "Antes vendia produtos para ajudar nas contas de casa; nem isso agora consigo fazer" (P1) \\
\hline & "Adoeci e me aposentei" (P2) \\
\hline & "Aposentada devido à DPOC, trabalhava em uma confecção; não tinha como continuar" (P5) \\
\hline \multirow{4}{*}{ Lazer } & "Não vou na praia, nada eu vou" (P1) \\
\hline & $\begin{array}{l}\text { "Tenho dificuldade de passear, fico cansando, faltando o ar, fica querendo faltar o ar na gente, } \\
\text { faltando o fôlego" (P2) }\end{array}$ \\
\hline & "Não tenho uma vida social" (P3) \\
\hline & "Deixei de me divertir, não danço mais" (P5) \\
\hline
\end{tabular}

Fonte: Dados da pesquisa, 2020.

Como nossas açôes sáo influenciadas por nossos valores, é essencial para o terapeuta ocupacional conhecer o valor que os pacientes colocam sobre as diferentes ocupaçóes, 
considerando o significado de desempenhar essas tarefas, assim como a dificuldades de realizálas, permitindo estabelecer as prioridades de intervenção, assim como identificar os resultados desejados por eles (Rogers \& Holm, 2002).

Neste sentido, na terapia ocupacional, ao considerar as habilidades e padrôes de desempenho e contexto na forma como essas ocupaçóes são realizadas e ao possibilitar o envolvimento dos pacientes com DPOC nessas ocupaçóes significativas, além de significado especial e valor, o desempenho dessas ocupaçóes pode produzir benefícios pessoais, sociais, econômicos para o paciente, suas famílias e a sociedade como um todo.

\section{Repercussóes da pandemia por COVID-19 no cotidiano do paciente com DPOC}

A principal medida preventiva preconizada na pandemia de COVID-19 para propagaçáo do vírus é o isolamento social, que acabou por alterar bruscamente a rotina de todas as pessoas, causando uma interrupção generalizada na vida ocupacional, atingindo todas as faixas etárias ou ciclos de vida em todo o mundo (Hammell, 2020).

Kamalakannan \& Chakraborty (2020) apontam que a pandemia restringiu o desempenho das ocupaçóes e que os pacientes com DPOC, por serem considerados mais vulneráveis, foram resguardados de realizar suas ocupaçóes significativas.

A seguir, os pacientes reconhecem as mudanças que sofreram na sua vida ocupacional e identificam o impacto dessa restrição social na pandemia.

O emocional sofri, tive crise de choro (P1).

Não saio de jeito nenhum, não vou nem na farmácia, nem na padaria (P2).

Faço as coisas dentro de casa; só ia para o tratamento aí no hospital; agora nem isso faço mais (P3).

Quebrou minha rotina (P4).

Ainda bem que tem o monitoramento pelo celular da equipe; porque senão eu nem sei (P5).

Antes da pandemia, fazia as compras com a minha filha, colocava no carrinho, mas quem carregava era minha filha; agora ela faz sozinha (P6).

Eu já era agradecida porque fazia parte do grupo Caminhada do Bosque; agora sou mais, porque estou recebendo todo o apoio pelo telefone e videos, mas sonho em voltar para participar das atividades (P7).

Gostava de ir para o tratamento e ver meus amigos na Caminhada do Bosque; sinto falta dai (P8).

Me sinto um passarinho engaiolado (P9).

A pandemia de COVID-19 destacou a necessidade humana de ocupação, sua complexidade e os efeitos da interrupção e privação ocupacional (Hammell, 2020). Os terapeutas ocupacionais reconhecem as mudanças que estáo ocorrendo na maneira como as pessoas realizam suas ocupaçóes como resultado da pandemia de COVID-19 e trabalharão 
com os pacientes para desenvolver estratégias para facilitar o acesso contínuo a suas ocupaçóes (World Federation of Occupational Therapy, 2020).

Logo, fornecer aos pacientes com DPOC atenção suficientemente cuidadosa é um verdadeiro desafio. É nossa responsabilidade manter e reforçar acompanhamento e gestão rigorosa desses pacientes, com o objetivo de limitar os efeitos colaterais que poderiam ser induzidos em decorrência da falta dessa assistência durante a pandemia (Deslée et al., 2020).

Muitos programas de reabilitaçâo pulmonar foram suspensos durante a pandemia para reduzir os riscos de disseminaçẫo da SARS-CoV-2, na qual foram introduzidas consultas remotas online, por vídeo ou telefone. Inclusive, essa foi a alternativa encontrada e utilizada para a continuidade da assistência aos pacientes com DPOC e outras pneumopatias acompanhados para tratamento no programa de manutenção pós reabilitação pulmonar - "A Caminhada do Bosque", na qual fazem parte os participantes da pesquisa.

O documento Global Initiative for Chronic Obstructive Lung Disease (2021) reconhece a necessidade de desenvolver novas abordagens para interagir com pacientes com DPOC e destaca as consultas remotas como uma excelente ferramenta para minimizar o risco de transmissão do coronavírus, assim como evitar o isolamento e a inatividade, evidenciando a importância dos pacientes permanecerem em contato com seus amigos, familiares e profissionais que os assiste por telecomunicaçáo e continuar ativos.

O Conselho Federal de Fisioterapia e Terapia Ocupacional (Brasil, 2020), por meio da resoluçấo n. o 516, de 20 de março de 2020, regulamentou a teleconsulta, o telemonitoramento e a teleconsultoria como modalidades viáveis de trabalho, a fim de permitir a continuidade de açóes para alguns grupos populacionais assistidos por terapeutas ocupacionais e fisioterapeutas.

Segundo Malfitano et al. (2020), a terapia ocupacional tem participado dessa alternativa de atendimento, tendo em vista a atenção do cotidiano dos sujeitos que, em seus diferentes modos de viver, têm em comum a necessidade de distanciamento social imposta pela pandemia de COVID-19.

O terapeuta ocupacional é o profissional capacitado para atuar frente à ruptura do cotidiano e busca, por meio de suas açôes, promover a reorganização de rotinas, a readaptação das atividades cotidianas e a ressignificação de sentidos diante da situaçấo e desafios atuais provocados pela pandemia de COVID-19 (De-Carlo et al., 2020).

\section{Consideraçóes Finais}

Com base na compreensão das dificuldades do paciente com DPOC em realizar suas ocupaçōes diárias, levando em consideração suas próprias experiências nas mais diversas situaçoes que fazem parte do seu cotidiano, foi possível conhecer o quanto a DPOC repercute no desempenho ocupacional e interfere nas mais diversas ocupaçóes que trazem significado e propósito à vida. Com exceção da educação e o brincar, todas as outras categorias que fazem parte do domínio ocupação foram mencionadas no estudo. Além das AVDs identificadas mobilidade funcional, banhar-se, higiene pessoal, vestir-se e atividade sexual - e AIVDs estabelecimento e gerenciamento do lar e o fazer compras -, outras ocupaçóes foram destacadas, como sono, trabalho, participação social e o lazer.

As ocupaçôes dos pacientes já limitadas pela DPOC foram ainda mais impactadas com as medidas restritivas causadas pela COVID-19. Ofertar uma assistência supervisionada por meio do atendimento remoto pode ser um excelente recurso utilizado pelo terapeuta ocupacional 
para ajudar os pacientes com DPOC a superar suas dificuldades e limitaçóes nesse período de pandemia.

Espera-se que novos estudos sejam instigados a serem realizados com foco nos aspectos relacionais e subjetivos, sob o ponto de vista paciente em relaçáo às suas limitaçóes em realizar suas atividades cotidianas em decorrência da DPOC.

\section{Referências}

American Occupational Therapy Association - AOTA. (2020). Occupational therapy practice framework: domain and process. The American Journal of Occupational Therapy, 74(Supl. 2), 1-87. PMid:34780625.

http://dx.doi.org/10.5014/ajot.2020.74S2001.

Bardin, L. (2009). Análise de conterido. Lisboa: Ediçōes 70.

Brasil. Conselho Federal de Fisioterapia e Terapia Ocupacional - COFFITO. (2006, 19 de julho). Resoluçâo COFFITO no 316/2006, de 19 de julho de 2006. Dispóe sobre a prática de atividades de vida diária, de atividades instrumentais da vida diária e tecnologia assistiva pelo Terapeuta Ocupacional e dá outras providências. Diário Oficial [da] República Federativa do Brasil, Brasília.

Brasil. Conselho Federal de Fisioterapia e Terapia Ocupacional - COFFITO. (2020, 20 de março). Resoluçáo no 516, de 20 de março de 2020. Dispóes sobre Teleconsulta, Telemonitoramento e Teleconsultoria. Diário Oficial [da] República Federativa do Brasil, Brasilia.

Caldas, A. S. C., Facundes, V. L. D., \& Silva, H. J. (2011). O uso da Medida Canadense de Desempenho Ocupacional em estudos brasileiros: uma revisão sistemática. Revista de Terapia Ocupacional da Universidade de São Paulo, 22(3), 238-244. http://dx.doi.org/10.11606/issn.2238-6149.v22i3p238-244.

De-Carlo, M. M. R. P., Gomes-Ferraz, C. A., Rezende, G., Buin, L., Moreira, D. J. A., Souza, K. L., Sacramento, A. M., Santos, W. A., Mendes, P. V. B., \& Vendrusculo-Fangel, L. M. (2020). Diretrizes para a assistência da terapia ocupacional na pandemia da COVID-19 e perspectivas pós-pandemia. Medicina, 53(3), 332-369. http://dx.doi.org/10.11606/issn.2176-7262.v53i3p332-369.

Deslée, G., Zysman, M., Burgel, P. R., Perez, T., Boyer, L., Gonzalez, J., \& Roche, N. (2020). Chronic obstructive pulmonary disease and the COVID-19 pandemic: reciprocal challenges. Respiratory Medicine and Research, 78, 1-4. http://dx.doi.org/10.1016/j.resmer.2020.100764.

Global Initiative for Chronic Obstructive Lung Disease - GOLD. (2021). Global strategy for the diagnosis, management, and prevention of COPD. Barcelona: GOLD.

Gulart, A. A., Santos, K. D., Munari, A. B., Karloh, M., Cani, K. C., \& Mayer, A. F. (2015). Relaçáo entre a capacidade funcional e a percepção de limitação em atividades de vida diária de pacientes com DPOC. Fisioterapia e Pesquisa, 22(2), 104-111. http://dx.doi.org/10.590/1809-2950/12836522022015.

Hagedorn, R. (2003). Fundamentos para a prática em terapia ocupacional. São Paulo: Roca.

Hammell, K. W. (2020). Engagement in living during the COVID-19 pandemic and ensuing occupational disruption. Occupational Therapy Now, 22, 7-8.

Kamalakannan, S., \& Chakraborty, S. (2020). Occupational therapy: the key to unlocking locked-up occupations during the COVID-19 pandemic. Wellcome Open Research, 5, 153. PMid:32766458. http://dx.doi.org/10.12688/wellcomeopenres.16089.1.

Kim, H. C., Mofarrahi, M., \& Hussain, S. N. (2008). Skeletal muscle dysfunction in patients with chronic obstructive pulmonary disease. International Journal of Chronic Obstructive Pulmonary Disease, 3(4), 637-658. PMid:19281080. http://dx.doi.org/10.2147/copd.s4480.

Machado, M. D. G. R. (2018). Bases da fisioterapia respiratória: terapia intensiva e reabilitaçäo. Rio de Janeiro: Guanabara Koogan.

Malfitano, A. P. S., Cruz, D. M. C., \& Lopes, R. E. (2020). Terapia ocupacional em tempos de pandemia: seguridade social e garantias de um cotidiano possível para todos. Cadernos Brasileiros de Terapia Ocupacional, 28(2), 401-404. http://dx.doi.org/10.4322/2526-8910.ctoED22802.

Minayo, M. D. S. (2014). O desafio do conhecimento: pesquisa qualitativa em saúde. São Paulo: HUCITEC.

Rabahi, M. F. (2013). Epidemiologia da DPOC: enfrentando desafios. Revista Pulmão, 22(2), 4-8. 
Rabe, K. F., \& Watz, H. (2017). Chronic obstructive pulmonary disease. Lancet, 389(10082), 1931-1940. PMid:28513453. http://dx.doi.org/10.1016/S0140-6736(17)31222-9.

Rogers, J. C., \& Holm, M. B. (2002). Avaliaçao das áreas de desempenho ocupacional. In M. Neistadt \& E. B. Crepeau (Eds.), Willard \& Spackman: terapia ocupacional (pp. 167-201). Rio de Janeiro: Guanabara Koogan.

Roth, G. A., Abate, D., Abate, K. H., Abay, S. M., Abbafati, C., Abbasi, N., \& Borschmann, R., and the GBD 2017 Causes of Death Collaborators. (2018). Global, regional, and national age-sex-specific mortality for 282 causes of death in 195 countries and territories, 1980-2017: a systematic analysis for the Global Burden of Disease Study 2017. Lancet, 392(10159), 1736-1788. PMid:30496103. http://dx.doi.org/10.1016/S01406736(18)32203-7.

Silva, A. L. G. D., Garmatz, E., Goulart, C. D. L., Carvalho, L. L., Cardoso, D. M., \& Paiva, D. N. (2017). Handgrip and functional capacity in Chronic Obstructive Pulmonary Disease patients. Fisioterapia em Movimento, 30(3), 501-507. http://dx.doi.org/10.1590/1980-5918.030.003.ao08.

Silva, C. M. S., Jesus, J. G. R., Cunha, E. F. S., \& Machado, A. S. (2015). Avaliaçáo da atividade de vida diária no paciente com doença pulmonar obstrutiva crônica (DPOC). Revista de Ciências Médicas e Biológicas, 14(3), 267-273. http://dx.doi.org/10.9771/cmbio.v14i3.14976.

Vaes, A. W., Delbressine, J. M. L., Mesquita, R., Goertz, Y. M. J., Janssen, D. J. A., Nakken, N., Franssen, F. M. E., Vanfleteren, L. E. G. W., Wouters, E. F. M., \& Spruit, M. A. (2019). Impact of pulmonary rehabilitation on activities of daily living in patients with chronic obstructive pulmonary disease. Journal of Applied Physiology, 126(3), 607-615. PMid:30496707. http://dx.doi.org/10.1152/japplphysiol.00790.2018.

Vaes, A. W., Wouters, E., Franssen, F., Uszko-Lencer, N., Stakenborg, K., Westra, M., Meijer, K., Schols, A., Janssen, P. P., \& Spruit, M. A. (2011). Task-related oxygen uptake during domestic activities of daily life in patients with COPD and healthy elderly subjects. Chest, 140(4), 970-979. PMid:21415128. http://dx.doi.org/10.1378/chest.10-3005.

World Federation of Occupational Therapy - WFOT. (2020). Public statement: occupational therapy response to the COVID-19 pandemic. Recuperado em 8 de março de 2021, de https:/www.wfot.org/assets/resources/WFOT-Public-Statement-Occupational-Therapy-Response-to-theCOVID-19-Pandemic.pdf

\section{Contribuiçáo dos Autores}

Roberta Fontenele de Vasconcelos: Coleta, organização, transcrição e análise das entrevistas. Marília Ximenes Freitas Frota: Orientação e revisão textual. Valéria Barroso de Albuquerque: Revisão crítica final. Marilene Calderaro da Silva Munguba: Revisão crítica final.

Todos os autores aprovaram a versão final do texto.

\section{Autor para correspondência}

Roberta Fontenele de Vasconcelos

e-mail: robertafontenele10@gmail.com

\section{Editora de seçáo}

Profa. Dra. Iza Faria-Fortini 